\title{
Rare Association of Sensorimotor Polyneuropathy and Klinefelter Syndrome (47,XXY): Case Report
}

\author{
E. Kararizou ${ }^{a} \quad$ A.F. Mentis $^{a} \quad$ K. Gkiatas $^{\text {b }} \quad$ P. Davaki ${ }^{a}$ \\ ${ }^{a}$ Department of Neurology, Aeginition Hospital, University of Athens School of Medicine, and \\ ${ }^{b}$ Department of Neurology, 251 Air Force Hospital, Athens, Greece
}

\section{Key Words}

47, XXY $\cdot$ Klinefelter syndrome $\cdot$ Peripheral polyneuropathy

\begin{abstract}
Objectives: To describe a case with the rare association of Klinefelter syndrome $(47, \mathrm{XXY})$ and peripheral sensorimotor polyneuropathy. Clinical Presentation and Intervention: A 50-year-old man with Klinefelter syndrome was referred to our neurology clinic complaining of pain, numbness and tingles in both legs, which began 10 years prior to admission. Two years before admission, the patient had difficulty in walking with progressive weakness. Conclusion: This report shows a patient with diagnosed Klinefelter syndrome, in whom symmetrical sensorimotor polyneuropathy developed in late adulthood.

Copyright $\odot 2011$ S. Karger AG, Basel
\end{abstract}

\section{Introduction}

Klinefelter syndrome refers to a group of chromosomal disorders in which there is at least an extra X-chromosome added to a normal karyotype 46,XY. However, the classic form of this syndrome is the one in which there is only an extra copy of the X-chromosome, resulting in the

\section{KARGER}

Fax +4161306 1234

E-Mail karger@karger.ch

www.karger.com
(C) 2011 S. Karger AG, Basel

1011-7571/11/0205-0480\$38.00/0

Accessible online at:

www.karger.com/mpp karyotype XXY. Other forms include 48, XXYY, 48, XXXY and 49,XXXXY [1]. The diagnosis is usually made during adolescence or early adulthood in males presenting with small testes with gynecomastia and hypergonadotropic hypogonadism. Characteristics of these syndromes are: infertility, small testes, decreased facial hair, gynecomastia, decreased pubic hair, a small penis and a tall body habitus. In parallel with this, in adulthood they present with loss of libido, decreased muscle bulk and tone, and an increased risk of mortality from diabetic complications $[1,2]$.

Neurological characteristics include: language-based learning disorders (80\%), difficulties with planning and inhibitory control, deficits in short-term auditory memory and auditory processing, as well as motor delay with truncal hypotonia and motor planning deficits [3].

Here, we present the case of a patient diagnosed with Klinefelter syndrome $(47, \mathrm{XXY})$ that was associated with polyneuropathy.

\section{Case Report}

A 50-year-old man referred to the Neurology Clinic, Athens University, complained of pain, numbness and tingles in both sides distally in the feet, which had commenced 10 years prior to admission. A progressive expansion of the inferior surface of the 
calf and then in the whole area of the lower extremity was observed. Two years before admission, the patient had difficulty in walking with progressive weakness. The family history did not reveal any neurological conditions.

At the beginning of the physical examination, the patient did not reveal that he had Klinefelter syndrome, which was confirmed by a karyotype analysis showing a 47,XXY type. The patient was initially on testosterone therapy ( $135 \mathrm{mg}$ by depot injection every 3 weeks) for 3 years starting at the age of 27 years, but he had decided to stop because it made him aggressive.

Neurological examination revealed a symmetrical reduction in muscle power in both arms and legs with difficulty in walking mainly on the tips of the feet. Using the Medical Council Research scale (based on grade of 5): head extension and flexion $=5$, arm abduction, flexion and extension $=5$, wrist extension and flexion $=4$, finger extension $=4$, abductor pollicis brevis $=4$, first interosseus dorsalis $=3$, hip flexion and extension $=5$, knee flexion and extension $=4$, foot dorsiflexion $=3$, and foot plantarflexion $=2$. Atrophies were visible only in the small muscles of the distal leg. Deep tendon reflexes were symmetrically diminished $(1+)$ in the upper extremities and absent in both lower extremities. Plantar responses were flexor. There were no fasciculations or sphincter disturbances. Coordination was normal. Touch sensation was impaired in a glove-and-stocking distribution to the wrist and ankles. Vibrational and positional senses were normal. There were no cranial abnormalities. All other aspects of the neurological examination were normal.

Other causes of peripheral neuropathy - such as diabetes, sarcoidosis, leprosy, malignancy, connective tissue disease, toxic exposure, nutritional deficiency and family history of neuromuscular disorders - were excluded by several appropriate clinical and laboratory investigations.

Routine hematological tests and chest X-ray were normal. Complete serological tests - including erythrocyte sedimentation rate, C-reactive protein, complement 3 , complement 4 , anti-nuclear antibodies, anti-DNA antibodies, rheumatic factor, cryoglobulin and immunoelectrophoresis - were obtained and found to be normal. Serum and urine specimens were tested for monoclonal protein. Serum immunofixation electrophoresis was normal. Serum antibodies to myelin-associated glycoprotein, GM1 and GQ1b were not present. Glucose, glycosylated hemoglobin, 2-hour oral glucose tolerance test and laboratory investigations of CSF were normal. The CSF Venereal Disease Research Laboratory testing and Western blot for Lyme disease were negative. Only thyroid-stimulating hormone (TSH) values were altered in the first measurement, but this was not confirmed in consecutive measurements. The thyroid gland was not palpable, and the patient was clinically euthyroid. Additionally, the ultrasound of the thyroid gland was normal.

Electromyography was performed. Nerve conduction studies showed mildly decreased motor and sensory conduction velocities, with significantly reduced amplitude of the motor and sensory compound action potentials, without block or dispersion. There was electromyographic evidence of chronic denervation and signs of active denervation. On the basis of these results, a diagnosis of sensorimotor axonal polyneuropathy was made.

Biopsy of the sural nerve was performed. Histology revealed fiber loss. Axonal degeneration was the predominant feature. The immunohistochemical study was negative to antihuman polyclonal antibodies (IgA, IgG, IgM), C3 and fibrinogen. Morpho- metric study revealed significant loss of myelinated fibers $(2,433$ $\pm 1,245)$. The mean axonal diameter was decreased $(2.87 \pm$ $0.93 \mu \mathrm{m})$. The $\mathrm{g}$ ratio was markedly decreased $(0.42 \pm 0.11)$ and supported the histopathological findings of mainly axonal involvement. The histograms of fiber diameter distributions showed damage to all diameter fibers.

\section{Discussion}

We report the clinical case of a patient with diagnosed Klinefelter syndrome who was also affected by symmetrical sensorimotor polyneuropathy. Klinefelter syndrome is the most common sex chromosome disorder, and is usually associated with autoimmune diseases, osteoporosis, primary hypothyroidism, diabetes mellitus, breast and prostate cancer, and leukemia. Polyneuropathy may represent a potentially hypogonadic complication of Klinefelter syndrome or alternatively could be a coexisting morbidity. A previous study [4] has shown that hypogonadal men with diabetes mellitus and diabetic foot neuropathy clinically improved following administration of testosterone. However, testosterone deficiency may have adverse effects on nerves other than the cavernosal nerve, as Traish et al. [5] showed that castration reduced NOS-containing nerve fibers, innervating corpus cavernosum tissue, and lead to reduced thickness of the nerve sheath of the cavernosal nerve in castrated animals. Unfortunately, our patient was initially on testosterone therapy, but decided to stop because it made him aggressive.

Moreover, three diseases in which hypogonadism is present have been linked in case reports with polyneuropathy: (1) Rud syndrome linked with hypogonadism, mental retardation, retinitis pigmentosa and hypertrophic polyneuropathy [6]; (2) Möbius sequence connected with axonal peripheral neuropathy and hypogonadotropic hypogonadism, in which administration of pulsatile gonadotropin-releasing hormone led to alleviation of the symptoms [7], and (3) familial cerebellar ataxia associated with hypogonadism and sensorimotor axonal neuropathy [8].

Nevertheless, the neuropathy presented could potentially be linked to another cause known to cause such a clinical entity. Among the causes we have found in the literature, both diabetes mellitus and thyroid dysfunction are related to Klinefelter syndrome. In fact, approximately $40 \%$ of people with Klinefelter syndrome have had a diabetic glucose tolerance test [1]. However, all diabetesrelated tests were normal in our patient, ruling out the possibility of diabetes-induced neuropathy. We have dis- 
cussed and investigated all the possible causes of polyneuropathy, and seen that diabetes is a more frequent factor which may be a complication of Klinefelter syndrome.

The relationship of our study to the others cited here was explained at the beginning of this section. Parallel to this, it has recently been observed that men with Klinefelter syndrome have lower values of free T4 and lower free T4:free $\mathrm{T} 3$ ratios, even in the absence of differences in TSH, free T3, TSH:fT4 ratios, thyroid volumes, or the prevalence of thyroid antibodies [8]. However, despite the lack of assessment of thyroid antibodies and free levels of hormones in our client, thyroid disease could be more or less excluded [9].

Matsubara et al. [10] described benign neurogenic amyotrophy in a patient with Klinefelter syndrome as an independent type of motor neuron disease, and suggested that the $\mathrm{X}$-chromosome plays an important part in the biology of motor neurons.
Kilnefelter syndrome tends to be associated with autoimmune diseases and especially with connective tissue disorders [11]. Nevertheless, our extensive clinical examination and complete serological tests, as well as the findings from the nerve biopsy, were negative for systemic autoimmune connective tissue disease.

Another cause could be vasculitis, which has been linked with Klinefelter syndrome in a single case report [12]. However, were this the case, the biopsy would have been expected to present evidence of vasculitis or nerve ischemia.

\section{Conclusion}

This report showed a patient with diagnosed Klinefelter syndrome, in whom symmetrical sensorimotor polyneuropathy developed in late adulthood.

\section{References}

1 Visootsak J, Graham JM Jr: Klinefelter syndrome and other sex chromosomal aneuploidies. Orphanet J Rare Dis 2006;1:42.

-2 Chauhan AK, Katiyar BC, Misra S, Thacker AK, Singh NK: Muscle dysfunction in male hypogonadism. Acta Neurol Scand 1986;73: 466-471.

>3 Giedd JN, Clasen LS, Wallace GL, Lenroot RK, Lerch JP, Wells EM, Blumenthal JD, Nelson JE, Tossell JW, Stayer C, Evans AC, Samango-Sprouse CA: XXY (Klinefelter syndrome): pediatric quantitative brain magnetic resonance imaging case-control study. Pediatrics 2007;119:e232-e240.

-4 Kalinchenko S, Zemlyanoy A, Gooren LJ: Improvement of the diabetic foot upon testosterone administration to hypogonadal men with peripheral arterial disease: report of three cases. Cardiovasc Diabetol 2009;8:19.
5 Traish A: Androgens play a pivotal role in maintaining penile tissue architecture and erection: a review. Dev Neurosci 2001;23: 441-451.

-6 Larbrisseau A, Carpenter S: Rud syndrome: congenital ichthyosis, hypogonadism, mental retardation, retinitis pigmentosa and hypertrophic polyneuropathy. Neuropediatrics 1982;13:95-98.

-7 Kawai M, Momoi T, Fujii T, Nakano S, Itagaki Y, Mikawa H: The syndrome of Möbius sequence, peripheral neuropathy, and hypogonadotropic hypogonadism. Am J Med Genet 1990;37:578-582.

-8 Erdemoğlu AK, Akbostanci MC, Selçuki D: Familial cerebellar ataxia and hypogonadism associated with sensorimotor axonal polyneuropathy. Clin Neurol Neurosurg 2000;102:129-134.
-9 Bjørn AM, Bojesen A, Gravholt $\mathrm{CH}$, Laurberg P: Hypothyroidism secondary to hypothalamic-pituitary dysfunction may be part of the phenotype in Klinefelter syndrome: a case-control study. J Clin Endocrinol Metab 2009;94:2478-2481.

- 10 Matsubara S, Yoshino M, Takamori M: Benign neurogenic amyotrophy in Klinefelter's syndrome. J Neurol Neurosurg Psychiatry 1994;57:640-642.

11 Rovenský J, Imrich R, Lazúrová I, Payer J: Rheumatic diseases and Klinefelter's syndrome. Ann NY Acad Sci 2010;1193:1-9.

12 Kim W, Sequeira W: Vasculitis in Klinefelter's syndrome. Ann Rheum Dis 1992;51: 1266-1267. 